\title{
Relationship between US and Brazilian ethanol prices: new evidence based on fractal regressions
}

Derick David Quintino (D), Department of Economics, Administration and Sociology, University of São Paulo, Piracicaba, Brazil

Ana Cantarinha, Instituto Politécnico de Beja, Beja, Portugal; CIMA - Center for Research on Mathematics and Its Applications, University of Évora, Évora, Portugal; CMA-Center of Mathematics and Applications, FCTNOVA, NOVA University of Lisbon, Lisbon, Portugal

Paulo Jorge Silveira Ferreira, VALORIZA-Research Center for Endogenous Resource Valorization, Portalegre, Portugal; Instituto Politécnico de Portalegre, Portalegre, Portugal; CEFAGE-UE, IIFA, Universidade de Évora, Évora, Portugal

Received November 14 2020; Revised December 24 2020; Accepted January 12 2021; View online at Wiley Online Library (wileyonlinelibrary.com); DOI: 10.1002/bbb.2192; Biofuels, Bioprod. Bioref. (2021)

\begin{abstract}
Two different fractal regression methodologies were employed with the aim of understanding the relationship between prices of ethanol produced in the USA and Brazil, the most important producers of ethanol. These methodologies, which have the advantage of giving us information about the relationships between different timescales, could also provide interesting and important information about this particular market. Our results suggest, first, some differences in the basic price in these markets, and second, a positive relationship between prices (as expected) but not a one-to-one relationship. (c) 2021 Society of Chemical Industry and John Wiley \& Sons, Ltd
\end{abstract}

Keywords: detrended cross-correlation analysis; detrending moving-average cross-correlation analysis; ethanol prices; fractal regressions

\section{Introduction}

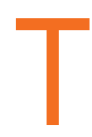
he present work aims to analyze the relationship between US and Brazilian ethanol prices. In 2019, the USA was the largest ethanol producer in the world, responsible for $54 \%$ of the supply, with Brazil in second place, with about $30 \%$ of global production. ${ }^{1}$ Jointly, both countries accounted for about $84 \%$ of the world's supply, implying that the price behavior in USA and Brazil largely determines the dynamics of the global ethanol market.
To analyze the degree of relationship between the prices, we employ a new methodology of fractal regressions, developed by Kristoufek ${ }^{2}$ and used by Ferreira and Kristoufek ${ }^{3}$ and Ferreira and Kristoufek. ${ }^{4}$ This is a robust methodology with the advantage of allowing analysis in various timescales. As highlighted by Caro et al., ${ }^{5}$ there is growing interest in the literature in understanding the spread behavior between West Texas Intermediate (WTI) and Brent oil indices, which is based on risk management, especially after the beginning of price divergence at the end of 2010. However, in the case of 
ethanol, which is our focus here, literature on the interrelated dynamics of these prices is still incipient.

American ethanol is largely derived from corn, whereas Brazilian ethanol comes almost entirely from sugarcane. Brazilian sugar, which like ethanol is also a by-product of sugarcane, is already a consolidated commodity in the international market, and Brazil's influence in the formation of international sugar prices has already been covered extensively in the literature. ${ }^{6}$ However, in relation to ethanol, the scenario is different, considering that it is a product that has been developed to a significant extent in Brazil and the USA only in recent decades.

Despite the importance of both countries in the global supply of ethanol, recent studies have focused only on their local markets, analyzing the dynamics of ethanol prices. ${ }^{7-12}$ Ethanol markets in Brazil and the USA are interconnected due to trade (Debnath et al., ${ }^{13}$ 2017), and $90 \%$ of imported Brazilian ethanol comes from the USA (Castañeda-Ayarza and Godoi, ${ }^{14}$ 2020), implying that deeper analysis of the topic is relevant, to understand the connections between the markets.

To the best of our knowledge, one of the first studies to investigate the possible relationship between ethanol prices in Brazil and the USA was by Quintino and David, ${ }^{15}$ where the authors verified the possibility of the ethanol futures contracts on the Chicago Mercantile Exchange (CME) playing a role in cross-hedging of Brazilian ethanol prices, compared to other important commodities such as gasoline and sugar.

More recently, Dutta ${ }^{11}$ found that ethanol markets move together in ways that cannot be considered regionalized. Hernandez et al. ${ }^{12}$ complemented the analysis and found evidence that, in the short term, the markets are not connected, but in the long term these markets are globalized, with mutual influence. Recently, Bouri et al. ${ }^{16}$ studied whether structural breaks are relevant for the prediction of ethanol market volatility, in the case of the USA, concluding that this was the case.

To study how ethanol prices are related, we will therefore consider this relationship using the fractal regression method, which is an important development in relation to the traditional OLS regression method. The main advantages of using fractal regressions lie in the possibility of estimating such relationships, even between non-stationary variables. If this non-stationarity is verified, the use of traditional OLS regressions could lead to spurious estimations. In the context of non-stationary time series, cointegration analysis is an alternative to obtain consistent estimators, although the inference is not direct. In the absence of cointegration, an error-correction model is another alternative, although this uses the first difference of the variables, which gives us different results (for more details, see Wooldridge). ${ }^{17}$ Moreover, the use of fractal regressions could provide information about the relationship between variables for different timescales and not only in a contemporary way, making it advantageous even if variables are stationary. Our investigation seeks to advance the analysis of Dutta ${ }^{11}$ and Hernandez et al. ${ }^{12}$ based on this new methodological approach.

This text describes the data and methodology, analyzes the results, and presents some final considerations.

\section{Methods and data}

Our sample was taken from June 2006 to June 2020, using weekly ethanol prices, with a total of 709 observations. Weekly prices were used to allow a direct comparison of the results with Dutta ${ }^{11}$ and Hernandez et al., ${ }^{12}$ who also used weekly data. Brazilian prices were retrieved from the Center for Advanced Studies on Applied Economics (CEPEA), linked to Luiz de Queiroz College of Agriculture at the University of Sao Paulo, and US prices were retrieved from Thomson Reuters Datastream.

Figure 1 depicts the evolution of both prices over time, revealing the similarities of their general behaviour. After a sharp drop in the price of American ethanol at the beginning of the sample, prices seem to move together. However, there are two moments of notable divergence with sudden price changes. First, in 2011, there was strong growth in the price of Brazilian ethanol for several reasons: strong growth in demand, high sugar prices and the costs of expanding sugar cane production, adverse weather conditions, appreciation of the exchange rate, and government policies for sugar in India and ethanol in

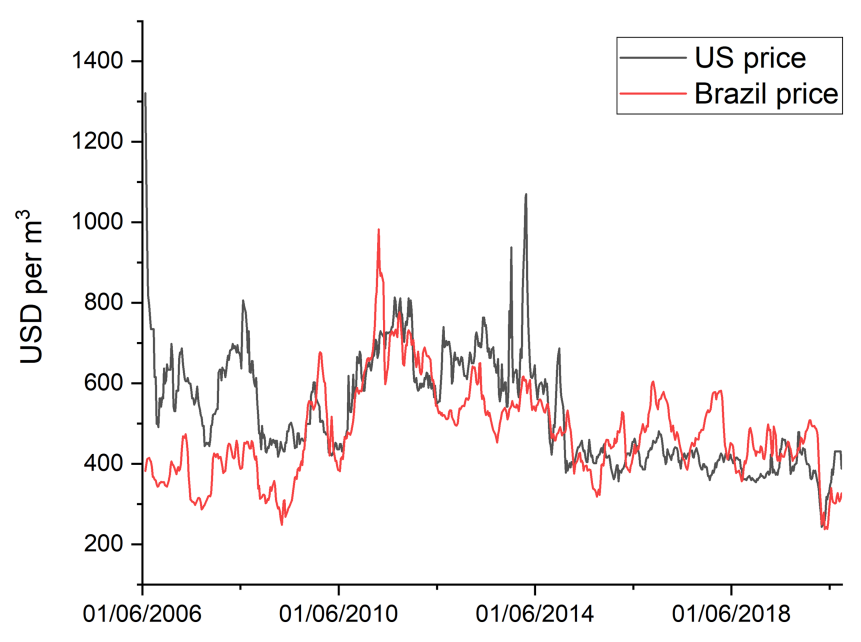

Figure 1. Evolution of US and Brazilian ethanol prices $\left(\mathrm{US} \$ / \mathrm{m}^{3}\right)$. 
Brazil. ${ }^{18}$ In the case of the USA, between 2012 and 2013, there was a major increase in corn prices, which implied a sharp increase in American ethanol prices. ${ }^{19}$

For a better understanding of the relation between ethanol prices in the USA and Brazil, we applied the fractal regression method. These regressions are based on other methods used to analyze fractal properties, namely the detrended fluctuation analysis (DFA) (proposed by Peng et al.), ${ }^{20}$ the detrending moving average (DMA) (Vandewalle and Ausloos), ${ }^{21}$ the detrended cross-correlation analysis (DCCA) (Podobnik and Stanley), ${ }^{22}$ and the detrending moving-average cross-correlation analysis (DMCA) (Arianos and Carbone, ${ }^{23} \mathrm{He}$ and Chen). ${ }^{24}$ These methodologies, combined in pairs, led to the creation of two different correlation coefficients: one based on the relationship between DFA and DCCA ( $\rho D C C A$ ) proposed by Zebende), ${ }^{25}$ and the other based on the relationship between DMA and DMCA ( $\rho$ DMCA) (proposed by Kristoufek). ${ }^{26}$ Over time, these methods showed their robustness regarding some features of time series, namely the existence of persistence, short-range correlations, structural breaks, or the existence of heavy tails, ${ }^{27,28}$ which are conditions present in financial time series, making this one of the research fields where those measures were used. Another interesting feature of these methodologies is their ability to capture the proposed relationships even in the context of non-stationary time series.

Based on these features, Kristoufek ${ }^{29,30}$ proposed the use of these measures in a regression-based methodology, transforming the relationship between DFA/DCCA and DMA/DMCA in fractal regressions, with estimators assessing the effects between two variables for different timescales, even in the presence of long-term memory and / or non-stationarity (an advantage, for example, when compared to the traditional ordinary least squares, OLS, which in those conditions could create spurious regressions).

Construction of the fractal regressions is similar to the OLS, although based on the ratio of the covariance of time series and the respective standard deviations of the pair DFA/ DCCA and DMA/DMCA, for a given scale - see Eqn (1):

$$
\begin{gathered}
\hat{\beta}^{D F A}(s)=\frac{F_{X Y, D F A}^{2}(s)}{F_{X, D F A}^{2}(s)} \\
\hat{\beta}^{D M A}(\lambda)=\frac{F_{X Y, D M A}^{2}(\lambda)}{F_{X, D M A}^{2}(\lambda)}
\end{gathered}
$$

with $F_{X, D F A}^{2}(s), F_{X Y, D F A}^{2}(s), F_{X, D M A}^{2}(\lambda), F_{X Y, D M A}^{2}(\lambda)$ being the covariance and variance functions of DFA/DCCA and DMA/DMCA procedures, respectively. These relationships allow estimating $\beta$ s, with the usual interpretations, for the specific scales, differentiating between short- and long-run behavior, and corresponding to lower or higher scales. In fact, multiscale approaches, like the ones proposed here, allow different interpretations for different timescales. Our estimations start with a timescale of 4 weeks (about 1 month) and extend to a timescale of 178 weeks (more than 3 years, distinguishing between short- and long-run results). More details about the methods and respective testing procedures can be found in the original papers by Kristoufek ${ }^{29,30}$ or in the empirical applications of Kristoufek, ${ }^{2}$ Ferreira and Kristoufek, ${ }^{3,4}$ or Kristoufek and Ferreira. ${ }^{31}$

\section{Results}

To assess the relationship between US and Brazilian ethanol prices, we estimated the following regression (Eqn (2)):

$$
\ln \left(\text { US }_{\text {price }_{t}}\right)=\beta_{0}+\beta_{1} \ln \left(B R_{\text {price }_{t}}\right)+u_{t}
$$

with $\beta_{0}$ and $\beta_{1}$ as the parameters of interest and $u$ being the usual error of a regression. In this case $\beta_{0}$ can be interpreted as the spread between the American and Brazilian price, while the value of $\beta_{1}$ is an elasticity measure of the relationship between the prices.

We started by applying the unit root tests, to confirm the existence or not of stationarity of the variables. We used the usual augmented Dickey Fuller test (see Wooldridge) ${ }^{17}$ and the Clemente $\mathrm{et} \mathrm{al.} .^{32}$ test, the latter allowing for the existence of structural breaks. The results, presented in Table 1, allow us

\begin{tabular}{|c|c|c|c|c|c|c|}
\hline \multirow[t]{3}{*}{ Variable } & \multicolumn{4}{|c|}{ Clemente, Montañes, and Reyes test } & \multicolumn{2}{|c|}{ Augmented DF test } \\
\hline & \multicolumn{2}{|c|}{ AO test } & \multicolumn{2}{|c|}{ IO test } & Without trend & With trend \\
\hline & t-stat & Lags & t-stat & Lags & t-stat & t-stat \\
\hline In (US price $\left._{t}\right)$ & -3.80 & 11 & -5.11 & 7 & -2.71 & -3.40 \\
\hline In $\left(B R_{\text {pricet }}\right)$ & -3.22 & 10 & -4.01 & 5 & -2.80 & -2.69 \\
\hline Critical value (5\% sig. Level) & -3.56 & & -4.27 & & -2.86 & -3.41 \\
\hline
\end{tabular}
to conclude that at a $5 \%$ significant level, the Brazilian price

\section{Table 1. Results of unit root tests.}


is non-stationary, while the US one presents mixed results (in the ADF test, the same lags of the AO test were used).

Considering the unit root tests, it seems useful to have a method that allows analysis even in the presence of nonstationary time series, as the proposed methods. The results of the estimations are presented in Figs 2 and 3, respectively, for the constant of the regression and for the regressor. It is interesting to note that the results of the DMCA are more stable than those of the DCCA, because the former uses moving averages in its estimation. This has also been noted in

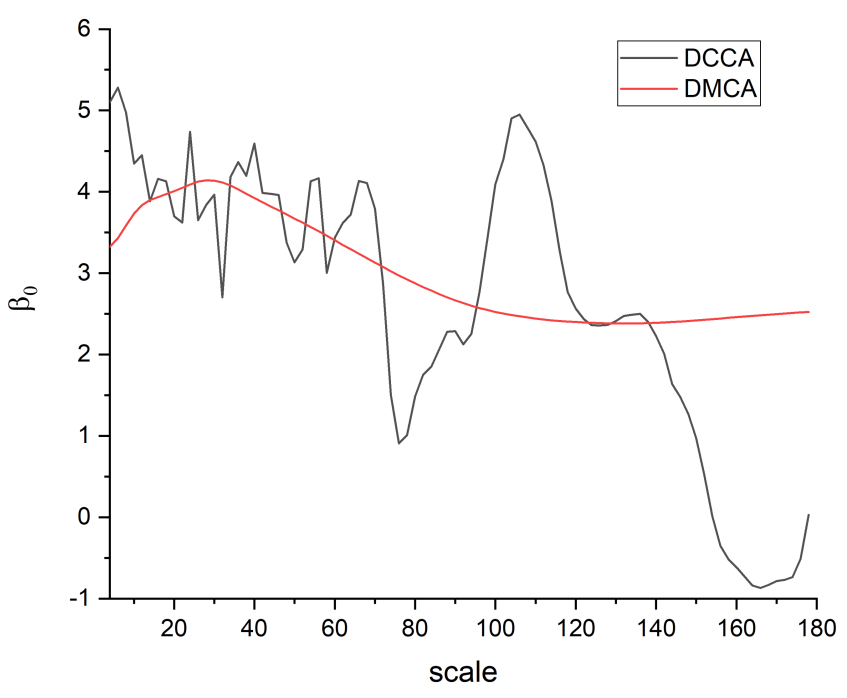

Figure 2. Estimation of the values of $\beta 0$ for the relationship between American and Brazilian ethanol prices, for DMA/ DMCA and DFA/DCCA.

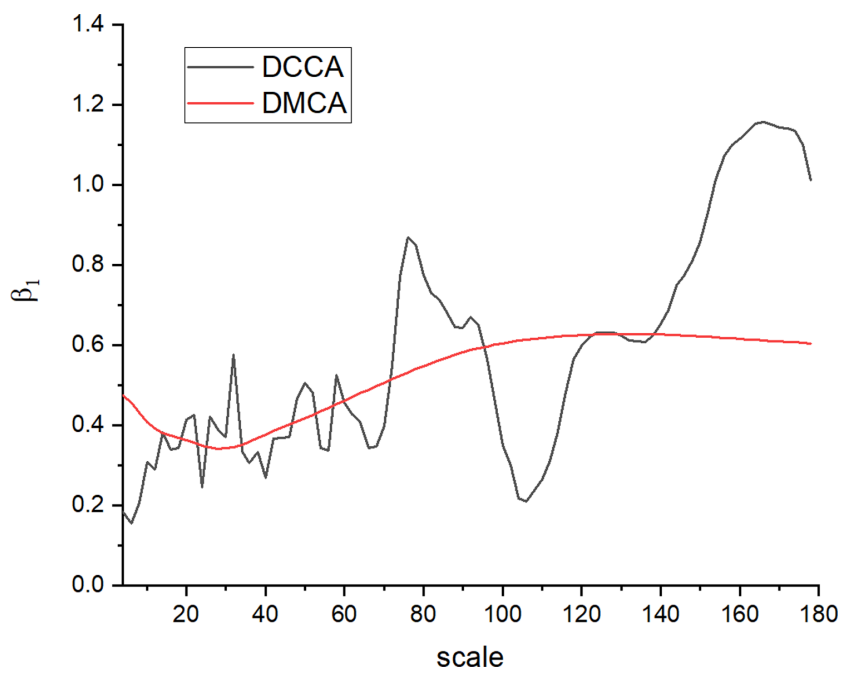

Figure 3. Estimation of the values of $\beta 1$ for the relationship between American and Brazilian ethanol prices, for DMA DMCA and DFA/DCCA. previous studies using both methodologies (see, for example, the empirical study mentioned at the end of the previous section' Presumably Kristoufek and Ferreira (note 31 ).

In relation to the coefficient $\beta_{0}$, which refers to the price spread, through the DMCA we find it reaches its peak in the scale of about 30 weeks. The lowest levels find some stability after 100 weeks. This means that the spread between prices is more evident in the short / medium run, stabilizing in the long run (over about 2 years). In relation to the DCCA, we observed reasonably oscillatory behavior around the curve generated by the DMCA, with increasing variability after approximately 70 weeks (this could be related to higher scales of the DCCA running with a lower number of observations, implying that the variability of the estimations increases).

Regarding $\beta_{1}$, which measures the intensity of the relationship between prices, in the case of the DMCA we have the lowest price relation strength around 30 weeks, with a coefficient of around 0.4 . The greatest strength of the price ratio occurs with the coefficient around 0.7 , in scales around 100 weeks. Once again, estimation through the DCCA has higher volatility, showing an increased connection in the long run (higher scales).

These facts may be linked to the seasonality of the two different raw materials from which ethanol originates: corn and sugar cane, respectively from the USA and Brazil. As highlighted by Murari et al., ${ }^{33}$ sugar cane production has cycles, and harvesting typically occurs in 12 months (52 weeks) or 18 months ( 80 weeks).

Our results have implications both for private agents' strategy (like investors and firms) and the design of public policies, in line with the results obtained by Dutta ${ }^{11}$ and Hernandez et al. ${ }^{12}$ In relation to the first group, the formulation of investment diversification will depend fundamentally on each agent's time horizon of interest, given that both the spread and the price elasticity vary over time. In this way, agents with more concentrated interests in the short term, where the connection between markets seems to be lower, will present different exposure from those interested in the medium or long term (when the parameter measuring the connection between both markets is higher). Furthermore, from the point of view of policy makers, and because of price transmission in the long run, this is relevant information for the design of possible policies, reacting to market events. For example, the existence of a given shock in a market, even a local shock, will affect prices in the other market. Finally, policies related to tariffs or to change export quotas, which have been the subject of recent negotiations, tend to affect the speed of price alignment (Castañeda-Ayarza and Godoi, 2020). ${ }^{14}$ 


\section{Conclusions}

This work aimed to investigate the ethanol price relationship between USA and Brazil, using a new fractal regression methodology, with two different approaches (DFA/DCCA and DMA/DMCA), which allows robust estimation even with non-stationary variables and taking into account various timescales, and not just in a contemporary analysis.

The results of the application of the DMCA method identify that the spread (i.e. B0), shows a decreasing trend as the timescale increases, meaning that the price difference tends to decrease. The same general movement is detected by the DCCA approach, although with greater volatility in the parameters. Regarding the coefficient measuring the linkage between prices, the coefficient is about 0.6 in the long run, without reaching the unitary level, meaning that, although positively related, the co-movements of the prices are not directly proportional. Once again, the DCCA coefficient shows higher volatility, depending on the timescales used, with the particularity of being near to 1 in the long run.

These findings are interesting and relevant for different agents, such as investors, policy-makers, and academics. For investors, our findings could help in the development of hedge strategies or portfolio diversification, and as these agents can be heterogeneous in terms of time preferences, fractal estimations provide estimates that are scale dependent with robustness. Regarding policy makers, according to Dutta ${ }^{11}$ and Hernandez et al., ${ }^{12}$ it is essential to have additional consistent evidence to determine whether prices are related - that is, whether the information flow that impacts the price of one market also affects others, or if prices behave independently, identifying markets that could be, respectively, globalized or regionalized. Depending on the nature of the relationship between prices, therefore, public policies must be oriented differently in order to achieve different goals. Finally, the study is also of academic interest by contributing to the debate on complex price interrelations bwtween the ethanol in different markets, even because of its relationship with the sustainable development.

Future research may shed light on this and other related issues in order to broaden understanding of ethanol price relationships, as new information accumulates and this market develops.

\section{Acknowledgements}

Derick Quintino wishes to acknowledge Coordenação de Aperfeiçoamento de Pessoal de Nível Superior - Brasil (CAPES) for funding support. This study was financed in part by (CAPES) - Finance Code 001. Paulo Ferreira also acknowledges the financial support of Fundação para a Ciência e a Tecnologia (grants UIDB/05064/2020 and UIDB/04007/2020).

\section{References}

1. RFA, Ethanol Industry Outlook. Renewable Fuels Association, Washington, DC (2019). https://ethanolrfa. org/wp-content/uploads/2020/02/2020-Outlook-Final-forWebsite.pdf [31 August 2020].

2. Kristoufek L, Fractality in market risk structure: Dow Jones industrial components case. Chaos Solitons Fract 110:69-75 (2018).

3. Ferreira $P$ and Kristoufek L, What is new about covered interest parity condition in the European Union? Evidence from fractal cross-correlation regressions. Phys $A$ 486:554566 (2017).

4. Ferreira $P$ and Kristoufek L, Uncovered interest rate parity through the lens of fractal methods: evidence from the European Union. Phys A 553:124257 (2020).

5. Caro JMB, Golpe AA, Iglesias J and Vides JC, A new way of measuring the WTI-Brent spread. Globalization, shock persistence and common trends. Energy Econ 85:104546 (2020).

6. Rumánková L, Smutka L, Maitah M and Benešová I, The interrelationship between sugar prices at the Main world sugar commodities markets. Sugar Technol 21:853-861 (2019).

7. Serra $T$ and Zilberman $D$, Biofuel-related price transmission literature: a review. Energy Econ 37:141-151 (2013).

8. Quintino DD, David SA and Vian CEDF, Analysis of the relationship between ethanol spot and futures prices in Brazil. Int J Finan Stud 5:11 (2017).

9. Capitani DHD, Junior JCC and Tonin JM, Integration and hedging efficiency between Brazilian and US ethanol markets. Contextus 16:93-117 (2018).

10. David SA, Inácio CMC Jr, Quintino DD and Machado JAT, Measuring the Brazilian ethanol and gasoline market efficiency using DFA-Hurst and fractal dimension. Energy Econ 85:104614 (2020).

11. Dutta A, Are global ethanol markets a 'one great pool'? Biomass Bioenergy 132:105436 (2020).

12. Hernandez JA, Uddin GS, Dutta A, Ahmed A and Kang SH, Are ethanol markets globalized or regionalized? Phys $A$ 551:124094 (2020).

13. Debnath D, Whistance $J$ and Thompson W, The causes of two-way US-Brazil ethanol trade and the consequences for greenhouse gas emission. Energy 141:2045-2053 (2017).

14. Castañeda-Ayarza JA and Godoi BA, Macro-environmental influence on the development of Brazilian fuel ethanol between 1975 and 2019. Renew Sustain Energy Rev 137:110457 (2020).

15. Quintino DD and David SA, Quantitative analysis of feasibility of hydrous ethanol futures contracts in Brazil. Energy Econ 40:927-935 (2013).

16. Bouri E, Dutta A and Saeed T, Forecasting ethanol price volatility under structural breaks. Biofuels Bioprod Biorefin 15(1):250-256 (2020).

17. Wooldridge JM, Introductory econometrics: A modern approach. 6th Edition, Cengage Learning, Boston, US (2016).

18. de Gorter H, Drabik D and Kliauga EM, Why ethanol prices are so high in Brazil. Biofuels 3:371-373 (2012).

19. Kristoufek $L$, Janda $K$ and Zilberman $D$, Comovements of ethanol-related prices: evidence from Brazil and the USA. Gcb Bioenergy 8:346-356 (2016). 
20. Peng CK, Buldyrev SV, Havlin S, Simons M, Stanley HE and Goldberger AL, Mosaic organization of DNA nucleotides. Phys Rev 49:1685-1689 (1994).

21. Vandewalle $\mathrm{N}$ and Ausloos $\mathrm{M}$, Multi-affine analysis of typical currency exchange rates. Eur Phys J 4:257-261 (1998).

22. Podobnik B and Stanley HE, Detrended cross-correlation analysis: a new method for analyzing two nonstationary time series. Phys Rev Lett 100:084102 (2008).

23. Arianos $S$ and Carbone $A$, Cross-correlation of long-range correlated series. J Stat Mech 2009:P03037 (2009).

24. He LY and Chen SP, A new approach to quantify power-law cross-correlation and its application to commodity markets. Phys A 390:3806-3814 (2011).

25. Zebende GF, DCCA cross-correlation coefficient: quantifying level of cross-correlation. Phys A 390:614-618 (2011).

26. Kristoufek L, Detrending moving-average cross-correlation coefficient: measuring cross-correlations between nonstationary series. Phys A 406:169-175 (2014).

27. Weron R, Estimating long-range dependence: finite sample properties and confidence intervals. Phys A 312:285-299 (2002).

28. Barunik J and Kristoufek L, On Hurst exponent estimation under heavy-tailed distributions. Phys A 389:3844-3855 (2010).

29. Kristoufek L, Detrended fluctuation analysis as a regression framework: estimating dependence at different scales. Phys Rev 91:022802 (2015).

30. Kristoufek L, Scaling of dependence between foreign exchange rates and stock Markets in Central Europe. Acta Phys Pol 129:908-912 (2016).

31. Kristoufek $L$ and Ferreira $P$, Capital asset pricing model in Portugal: evidence from fractal regressions. Portuguese Econ J 17:173-183 (2018).

32. Clemente J, Montañés A and Reyes M, Testing for a unit root in variables with a double change in the mean. Econ Lett 59(2):175-182 (1998).

33. Murari TB, Nascimento Filho AS, Pereira EJ, Ferreira P, Pitombo S, Pereira HB et al., Comparative analysis between hydrous ethanol and gasoline $\mathrm{C}$ pricing in Brazilian retail market. Sustainability 11:4719 (2019).

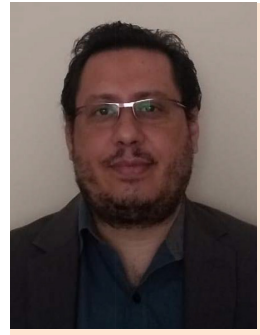

\section{Derick David Quintino}

Derick Quintino is currently a PhD candidate in applied economics at the University of São Paulo (Brazil) and his recent research has appeared in international peer-reviewed journals. His major interests are focused on agricultural and energy economics, including financial markets.

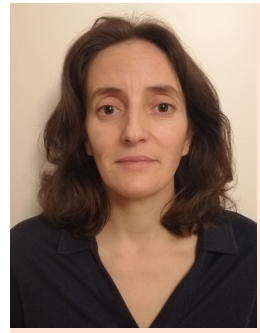

\section{Ana Cantarinha}

Ana Cantarinha is a professor at the Polytechnic Institute of Beja, with a master's degree in economics and a $\mathrm{PhD}$ in statistics. She is researcher at CMA (Research Center of Mathematics and its Applications New University of Lisbon) and CIMA (Research Center of Mathematics and its Applications University of Évora).

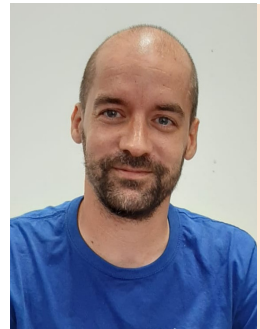

\section{Paulo Jorge Silveira Ferreira}

Paulo Ferreira has a PhD in management, and several peerreviewed published papers in international journals and technical books, focused mainly on the analysis of financial markets. He is a professor at the Polytechnic Institute of Portalegre and a researcher at VALORIZA - Research Center for Endogenous Resource Valuation. 